\title{
Localization of Latent Epileptic Activities Using Spatio-Temporal Independent Component Analysis of fMRI Data
}

\author{
Huafu Chen · Dezhong Yao • Guangming Lu • \\ Zhiqiang Zhang $\cdot$ Qiaoli Hu
}

Published online: 31 July 2007

(C) Springer Science+Business Media, LLC 2007

\section{Erratum to: Brain Topogr (2006) 19(1/2):21-28 DOI 10.1007/s10548-006-0009-7}

In the paper: "Localization of Latent Epileptic Activities Using Spatio-Temporal Independent Component Analysis of fMRI Data", that was recently published in Brain Topography Volume 19, Numbers 1/2, Winter 2006, pages 21-28, by Huafu Chen, Dezhong Yao, Guangming Lu, Zhiqiang Zhang and Qiaoli Hu, most of the first 3 paragraphs of the Introduction, and the final 2 paragraphs of the
Discussion and Conclusion, had been taken verbatim and without appropriate acknowledgement, from a paper (listed in the reference) in NeuroImage, 2004, 21: 473-481: "Resting fMRI with Temporal Clustering Analysis for Localization of Epileptic Activity Without EEG" by Victoria Morgan, Ronald Price, Amir Arain, Pradeep Modur and Bassel Abou-Khalil.

Brain Topography regrets that this incident has taken place, and steps have been taken to minimize any recurrence.

The online version of the original article can be found under doi: 10.1007/s10548-006-0009-7.

H. Chen $(\bowtie)$

School of Applied Mathematics, University of Electronic Science and Technology of China, Chengdu 610054, China e-mail: chenhf@uestc.edu.cn

D. Yao $\cdot$ Q. Hu

School of Life Science and Technology, University of Electronic Science and Technology of China, Chengdu 610054, China

G. Lu $\cdot$ Z. Zhang

Department of Medical Imaging, Nanjing General Hospital of PLA, Nanjing 210002, China 